\title{
Allicin depresses biological activities of pancreatic cancer cells by regulating miRNA-339-5p/ZNF-689 axis
}

\section{Type}

Research paper

\section{Keywords}

Allicin, PANC-1, Hs766T, ZNF-689, miRNA-339-5p

\begin{abstract}
Introduction

The aim of this study was to discuss Allicin's anti-tumor effects and relative in pancreatic cancer cell via vitro and vivo study.

Material and methods

Using PANC-1 and Hs766T cell as research cell lines in our study. The PANC-1 abd GS799T cell were respectively treated with difference concentrations of Allicin. In next step, the PANC-1 abd GS799T cell were transfected with si-miRNA-339-5p which knockdown miRNA-339-5p. The cells were treated with difference concentration of Allicin. Measuring cell proliferation, apoptosis, invasion and migration by MTT, EDU, flow cytometry, TUNEL, transwell and wound healing assay. Relative gene (miRNA-339-5p, ZNF-689, Caspase-3, Caspase-8, E-cadherin and Vimentin) and protein (ZNF-689, Caspase-3, Caspase-8, E-cadherin and Vimentin) expression were evaluated by RTqPCR and WB assay.
\end{abstract}

\section{Results}

Allicin had effects to suppress PANC-1 and Hs766T cell biological activities including cell proliferation, invasion and migration with cell apoptosis significantly increasing, however, with simiRNA-339-5p which inhibit miRNA-339-5p expression transfection, the cells biological activities enhancing with cell apoptosis depressing, Compared with NC group, miRNA-339-5p, Caspase-3, Caspase-8 and E-cadherin expression were significantly increased and ZNF-689 and Vimentin expression were significantly depressed in Allicin treated groups, however, with miRNA-339-5p inhibitor transfection, the Allicin's effects significantly disappear.

\section{Conclusions}

Allicin had anti-tumor effect to pancreatic cancer biological activities via regulation miRNA-339-5p/ZNF-689 axis in vitro study. 


\title{
Allicin inhibits the biological activities of pancreatic cancer cells by regulating the miRNA-339-5p/ZNF-689 axis
}

Running Title: Allicin and pancreatic cancer

\begin{abstract}
Introduction: The aim of this study was to characterise the anti-tumour effects of allicin in pancreatic cancer cells in vitro.

Materials and methods: PANC-1 and Hs766T human pancreatic cancer cell lines were treated with various concentrations of allicin. PANC-1 cells were also transfected with si-miRNA-339-5p which knocks down miRNA-339-5p expression. After treating the transfectants with various concentrations of allicin, we measured cell proliferation, apoptosis, invasion and migration by MTT, EDU, flow cytometry, TUNEL, Transwell and wound healing assays. Relative gene (miRNA-339-5p, ZNF-689, caspase-3,
\end{abstract}


caspase-8, E-cadherin and vimentin) and protein (ZNF-689, caspase-3, caspase-8, E-cadherin and vimentin) expression were evaluated by qRT-PCR and western blot analysis.

Results: Allicin inhibited PANC-1 and Hs766T cell biological activities, including proliferation, invasion and migration, with cell apoptosis significantly increasing. Following transfection, si-miRNA-339-5p inhibited the miRNA-339-5p expression and the biological activities of cells were enhanced, whereas apoptosis was inhibited. Compared with the normal control group, miRNA-339-5p, caspase-3, caspase- 8 and E-cadherin expression significantly increased, whereas ZNF-689 and vimentin expression significantly decreased in the allicin-treated groups. However, following miRNA-339-5p inhibitor transfection, the effects of allicin were significantly ameliorated.

Conclusion: Allicin exhibits anti-tumour effects on the biological activities of pancreatic cancer cells through regulation of the miRNA-339-5p/ZNF-689 axis in vitro.

Keywords: allicin; PANC-1; Hs766T; ZNF-689; miRNA-339-5p

\section{Introduction}

Pancreatic cancer is a common malignant tumour in humans. Recent statistics have revealed that the proportion of pancreatic cancer deaths ranks 4th among the ten major cancers in the United States in 2019 [1]. Because of the occult onset of pancreatic cancer, sensitive and specific markers are insufficient for its early diagnosis [2]. Most patients present with the advanced stage when diagnosed, which is accompanied by distant metastasis, and only $15 \%-20 \%$ of patients are eligible for surgery [3]. Therefore, for most patients, chemotherapy is the primary treatment available to prolong survival and improve the quality of life. Although progress has been made in treating pancreatic cancer in recent years, including the combined use of 5-fluorouracil, calcium folinate, irinotecan and oxaliplatin (FOLFIRINOX), and paclitaxel combined with gemcitabine, the overall prognosis remains poor [4]. The 
five-year survival rate for pancreatic cancer is still in the range of 3\%-9\% [1], so it is urgent to identify and develop new drugs to treat pancreatic cancer.

Allicin is a natural sulfur-containing compound extracted from garlic with a molecular formula of $\mathrm{C}_{6} \mathrm{H}_{10} \mathrm{OS}_{2}$. It primarily consists of diallyl sulfides, diallyl disulfides and diallyl trisulfides. Pharmacological studies have demonstrated that allicin can inhibit proliferation and induce apoptosis of various tumour cells [5-8]. However, studies on the effect of allicin on inhibiting the occurrence and development of pancreatic cancer are relatively limited. In this study, pancreatic cancer cell lines were treated with allicin at different doses in vitro to observe its effects. The expression of genes and proteins related to various signalling pathways were also evaluated.

MicroRNAs (miRNAs) represent a class of highly conserved endogenous non-coding RNAs that can silence genes at the post-transcriptional level by pairing with their corresponding target mRNAs. They play an important role in the aetiology and development of tumours [9]. Recent studies have shown that miRNA-339-5p has an important role in multiple tumour types [10-12]. Our previous results have also demonstrated that miRNA-339-5p overexpression can effectively inhibit various biological activities in pancreatic cancer [13]. However, the role of miRNA-339-5p in attenuating the effects of allicin in pancreatic cancer remains unclear.

\section{Materials and methods}

Cell lines and reagents

Standard allicin was purchased from Nanjing DASF Biotech Co., Ltd. (Jiangsu, purity $98.5 \%$ ). The pancreatic cancer cell lines PANC-1 and Hs766T were obtained from the Shanghai Institute of Cell Biology, Chinese Academy of Sciences. Trizol reagent was purchased from Invitrogen and the real-time fluorescent quantitative PCR kit was obtained from Takara. Reverse transcription, upstream and downstream primers, internal reference U6, exogenous si-miRNA-339-5p and an unrelated sequence (si-NC) were synthesised by the Nanjing KeyGEN Biotech Co., Ltd. 
Liposome-2000 was purchased from Invitrogen. The Transwell chambers and Matrigel were obtained from Millipore (USA) and BD (USA), respectively. ZNF-689, caspase-3, caspase-8, vimentin, E-cadherin and GAPDH antibodies were purchased from Abcam. The EDU staining and TUNEL kits were obtained from the Nanjing KeyGEN Biotech Co., Ltd.

Cell culture

PANC-1 and Hs766T cells were cultured in RPMI 1640 medium containing 10\% fetal bovine serum (FBS) at $37{ }^{\circ} \mathrm{C}$ and $5 \% \mathrm{CO}_{2}$. The experiments were carried out when the cells were in a logarithmic growth phase.

\section{Drug treatment}

When the cells grew to $70 \%-80 \%$ confluence, they were digested with trypsin and passaged once every $2-3 \mathrm{~d}$. Those in the logarithmic growth phase were collected. PANC-1 and Hs766T human pancreatic cancer cells were starved in serum-free medium for $12 \mathrm{~h}$ and treated with allicin $(5,10$ and $20 \mu \mathrm{g} / \mathrm{mL})$.

\section{Cell transfection}

One day prior to transfection, $2 \times 10^{5}$ cells were seeded into 6 -well plates and transfected when the cells had grown to approximately 50\% confluence. Exogenous si-miRNA-339-5p/si-NC and liposome-2000 (5 $\mu \mathrm{l})$ were combined and diluted with OPTIMEMI to a final volume of $250 \mu \mathrm{l}$. After mixing and standing, the suspension was added to the PANC-1 and Hs766T cells. After $6 \mathrm{~h}$, RPMI 1640 medium containing $10 \%$ FBS was added and the culture was continued.

\section{qRT-PCR assay}

After treatment for $48 \mathrm{~h}$, total RNA was extracted from each group of cells with Trizol and the concentration and purity were determined. PCR was done with miRNA-and gene-specific primers using SYBR Green I fluorescent dye detection. The internal reference for the miRNA was U6 and that of the other genes was 
GAPDH. The average values were used for calculations and the results were analysed using the comparative $\mathrm{Ct}$ method for relative quantification (2- $\triangle \triangle \mathrm{Ct}$ method). The relative expression of each gene was calculated and the experiments were repeated thrice. The sequences of the primers are provided in Table 1.

\section{MTT assay}

After treatment for $48 \mathrm{~h}, 10 \mu \mathrm{L}$ of $5 \mathrm{mg} / \mathrm{mL}$ MTT was added to each well. Four hours later, the culture medium was removed and discarded, and the reaction was terminated by adding $100 \mu \mathrm{L}$ of DMSO to each well. After placing in an oscillator for $10 \mathrm{~min}$, the absorbance values were measured at $570 \mathrm{~nm}$ using a microplate reader and the cell proliferation rates were calculated.

\section{Cell invasion assay}

After treatment for $48 \mathrm{~h}$, the cells in each group were digested with trypsin and centrifuged. The cells were resuspended to a density of $5 \times 10^{5}$ cells $/ \mathrm{ml}$ using serum-free RPMI 1640 medium. RPMI 1640 medium containing 10\% FBS was then added to the lower compartment of a Transwell chamber and $100 \mu$ of cell suspension was added to the upper compartment and incubated for $24 \mathrm{~h}$. Afterwards, the cells were treated with $1 \mathrm{ml}$ of $4 \%$ paraformaldehyde (PFA) at room temperature for $2 \mathrm{~min}$, followed by $1 \mathrm{ml}$ of anhydrous methanol at room temperature for $20 \mathrm{~min}$ and $1 \mathrm{ml}$ Giemsa solution in the dark at room temperature for $15 \mathrm{~min}$. After wiping off the cells on the inner surface of the bottom of the chamber, the invading cells were placed on a glass slide. Using a light microscope $(200 \times), 5$ visual fields were randomly selected to count the number of cells crossing the membrane. The experiment was repeated thrice.

\section{Cell migration assay}

After treatment for $48 \mathrm{~h}$, the cells in each group were digested with trypsin, collected and resuspended. The cells were diluted to $5 \times 10^{5}$ cells $/ \mathrm{ml}$ and seeded into 6-well plates for routine culture. When the cells reached $90 \%$ confluence, a vertical 
line was scratched at the bottom of the 6 -well plates with a $20 \mu$ pipette tip. After washing with phosphate buffer solution (PBS), a serum-free medium was added for routine culture. Sampling and imaging were done at $0 \mathrm{~h}$ and $48 \mathrm{~h}$. The experiment was repeated thrice.

\section{Western blot analysis}

After treatment for $48 \mathrm{~h}$, the cells in each group were collected and cell lysates were prepared. The total protein concentration was determined and the proteins were denatured by adding $1 \times$ loading buffer. Subsequently, 10\% SDS-PAGE was done using $20 \mu \mathrm{g}$ of each protein sample per well. The gels were transferred to PVDF membranes using a wet-transfer method and sealed in 5\% skim milk powder for $2 \mathrm{~h}$. Primary antibodies diluted with TBST at 1:1000 were added and incubated overnight at $4{ }^{\circ} \mathrm{C}$. Sheep anti-rabbit secondary antibody (1:5000 dilution) was added and the membranes were incubated at room temperature for $2 \mathrm{~h}$ followed by enhanced chemiluminescence detection. GAPDH was used as an internal reference. Relative protein expression was expressed as the ratio of the grey value of the target protein to that of GAPDH. The experiment was repeated thrice.

\section{Flow cytometry}

After treatment for $48 \mathrm{~h}$, the cells in each group were collected for apoptosis detection using an Annexin V-FITC staining kit. The cells were washed twice with PBS and an appropriate amount of trypsin was added to prepare a cell suspension. The supernatant was discarded after centrifugation. The cell suspension $(100 \mu \mathrm{g})$ was collected and combined successively with $500 \mu$ binding buffer and $5 \mu$ Annexin V-FITC, followed by incubation at room temperature in the dark for $5 \mathrm{~min}$. Then, 10 $\mu \mathrm{g}$ of $20 \mathrm{mg} / \mathrm{L}$ propidium iodide was added and the reactions were incubated in the dark for $15 \mathrm{~min}$. Within $1 \mathrm{~h}$, apoptosis detection was conducted using a flow cytometer and the results were analysed with the Cell Quest software. 
After treatment for $48 \mathrm{~h}$, the cells were digested with trypsin and counted using a cell counting chamber. Cells were seeded into 96-well plates at 8,000 cells/well with 3 duplicates for each group. After $24 \mathrm{~h}$, the culture medium was discarded and each well was treated with $100 \mu \mathrm{l}$ of $50 \mu \mathrm{M}$ EDU solution (diluted with complete cell culture medium at 1:1000) followed by incubation in a $5 \% \mathrm{CO}_{2}$ incubator at $37{ }^{\circ} \mathrm{C}$ for $2 \mathrm{~h}$. After the cells were washed once or twice with PBS, each well was treated with $50 \mu \mathrm{l}$ cell fixative (40 g/L PBS dissolved with PFA) and incubated at room temperature for $30 \mathrm{~min}$. The fixative was then discarded and each well was treated with $50 \mu 1$ of 2 $\mathrm{mg} / \mathrm{ml}$ glycine and incubated on a decolorising shaking table for $5 \mathrm{~min}$. After discarding the glycine and washing the cells with PBS, $100 \mu \mathrm{l}$ of penetrant (5 g/L PBS containing Triton X-100) was added to each well and incubated for $10 \mathrm{~min}$, followed by washing once with PBS. Subsequently, $100 \mu 11 \times$ Apollo solution was added to each well followed by incubation on a shaking table at room temperature in the dark for $30 \mathrm{~min}$. After discarding the Apollo solution, $100 \mu \mathrm{l}$ penetrant was added and washed thrice, and the penetrant was discarded. Finally, $1 \times$ Hoechst reaction solution (diluted with deionised water at 1:100) was added at $100 \mu \mathrm{l} /$ well and the plates were incubated at room temperature in the dark for $30 \mathrm{~min}$. Immediately after DNA staining, the cells were observed under an inverted fluorescence microscope and 5 fields of vision were randomly selected from each well for imaging.

\section{Detection of apoptosis by TUNEL assay}

After treatment for $48 \mathrm{~h}$, the culture medium in each group was removed, and the cells were washed once with PBS and fixed with $100 \mu \mathrm{l}$ of $4 \%$ PFA at room temperature for $30 \mathrm{~min}$. After washing thrice with PBS, PBS containing 0.3\% Triton $\mathrm{X}-100$ was added and incubated at room temperature for $5 \mathrm{~min}$. Then, the cells were washed with PBS thrice and TUNEL solution, prepared according to the manufacturer's (Beyotime) instructions, was added and incubated at $37{ }^{\circ} \mathrm{C}$ for $1 \mathrm{~h}$. The number of apoptotic cells was determined by fluorescence microscopy. 
Statistical analysis was done using the SPSS 22.0 software and the experimental results were repeated at least 3 times. The measured data were all in accordance with a normal distribution with homogeneous variance and were expressed as the mean \pm standard deviation. Comparison between two groups was made using a t-test and $P<$ 0.05 was considered statistically significant.

\section{Results}

Effect of allicin on the proliferation of pancreatic cancer cells

The results of the MTT assay (Figure 1A \& 1B) showed that when compared with the normal control (NC) group, cell proliferation was significantly inhibited in the allicin treatment groups (low, middle and high doses) $(P<0.05)$. EDU staining (Figure 1C \& 1D) revealed that compared with the NC group, the number of EDU-positive cells in the allicin treatment groups (low, middle and high doses) decreased significantly $(P<0.05)$. Significant differences were observed in the cell proliferation rate and the number of EDU-positive cells in the allicin treatment groups $(P<0.05$, Figure 1).

Effect of allicin on apoptosis of pancreatic cancer cells

Flow cytometry (Figure 2A \& 2B) revealed that the apoptotic rate of the allicin treatment groups (low, middle and high doses) was significantly higher compared with that of the NC group $(P<0.05)$. TUNEL staining results (Figure $2 \mathrm{C} \& 2 \mathrm{D}$ ) demonstrated that compared with the NC group, the number of positive apoptotic cells increased significantly in the allicin treatment groups (low, middle and high doses $)(P<0.05)$. After treatment with allicin at different concentrations, both the apoptotic rate and the number of positive apoptotic cells were significantly different among the allicin treatment groups $(P<0.05$, Figure 2$)$.

Effects of allicin on the invasion and migration of pancreatic cancer cells

Transwell assays (Figure 3A \& 3B) revealed that compared with the NC group, 
the number of invading cells in the allicin treatment groups (low, middle and high doses) decreased significantly $(P<0.05)$. The wound healing assay (Figure 3C \& 3D) revealed that the rate of wound healing in the allicin treatment groups (low, middle and high doses) was significantly lower than that in the $\mathrm{NC}$ group $(P<0.05$, respectively). After treatment with different concentrations of allicin, the number of invading cells and the rate of wound healing were significantly different among the allicin treatment groups $(P<0.05$, Figure 3$)$.

Effects of allicin on gene and protein expression

Quantitative RT-PCR and western blot results (Figure 4) revealed that compared with the NC group, the gene and protein expression of miRNA-339-5p, caspase-3, caspase- 8 and E-cadherin in the allicin treatment groups (low, middle and high doses) increased significantly, and the expression of ZNF-689, vimentin mRNA and protein also increased significantly $(P<0.05$, Figure 4$)$. After treatment with allicin at different concentrations, significant differences were observed in the expression of relevant genes and proteins among the allicin treatment groups $(P<0.05$, respectively, Figure 4).

Effect of miRNA-339-5p on the inhibition of proliferation by allicin in pancreatic cancer cells

The MTT assay (Figure 5A \& 5B) showed that compared with the NC group, the cell proliferation rate of the allicin group and the allicin + si-NC group decreased significantly $(P<0.001$, Figure 5A \& 5B). However, after transfection with si-miRNA-339-5p, the cell proliferation rate in the allicin + si-miRNA-339-5p group was significantly higher compared with that in the allicin group $(P<0.001$, Figure 5A $\& 5 B$ ). The results of EDU staining (Figure 5C \& 5D) indicated that compared with the NC group, the number of EDU-positive cells in the allicin and allicin + si-NC groups decreased significantly $(P<0.001$, Figure 5C \& 5D). After transfection with si-miRNA-339-5p, the number of EDU-positive cells in the allicin + si-miRNA-339-5p group increased significantly compared with the allicin group $(P<$ 
0.001, Figure 5C \& 5D).

Effect of miRNA-339-5p in the enhancement of apoptosis by allicin in pancreatic cancer cells

The results of flow cytometry (Figure 6A \& 6B) indicated that the apoptotic rate of the allicin group and the allicin + si-NC group was significantly higher than that of the NC group $(P<0.001$, Figure 6A \& 6B). However, after transfection with si-miRNA-339-5p, the apoptotic rate of the allicin + si-miRNA-339-5p group was significantly lower compared with the allicin group $(P<0.001$, Figure 6A \& 6B). TUNEL results (Figure 6C \& 6D) revealed that the number of positive apoptotic cells in the allicin group and the allicin + si-NC group increased significantly compared with the NC group $(P<0.001$, Figure 6C \& 6D). After transfection with si-miRNA-339-5p, the number of positive apoptotic cells in the allicin + si-miRNA-339-5p group was significantly lower than that in the allicin group $(P<$ 0.001 , Figure 6C \& 6D).

Effects of miRNA-339-5p on the inhibition of invasion and migration by allicin in pancreatic cancer cells

The Transwell assays (Figure 7A \& 7B) revealed that compared with the NC group, the number of invading cells in the allicin group and the allicin + si-NC group decreased significantly $(P<0.001$, Figure 7A \& 7B). However, after transfection with si-miRNA-339-5p, the number of invading cells in the allicin + si-miRNA-339-5p group increased significantly compared with that of the allicin group $(P<0.001$, Figure 7A \& 7B). The wound healing assay (Figure 7C \& 7D) demonstrated that compared with the NC group, the wound healing rate of the allicin group and the allicin + si-NC group decreased significantly $(P<0.001$, Figure 7C \& 7D). After transfection with si-miRNA-339-5p, the wound healing rate of the allicin + si-miRNA-339-5p group was significantly higher compared with that in the allicin group $(P<0.001$, Figure 7C \& 7D). 
Gene and protein expression analysis

Quantitative RT-PCR (Figure 8A \& 8B) revealed that compared with the NC group, the expression of miRNA-339-5p, caspase-3, caspase- 8 and E-cadherin RNA in the allicin group and the allicin + si-NC group increased significantly, whereas the expression of ZNF-689 and vimentin mRNA decreased significantly $(P<0.001$, Figure 8A \& 8B). After transfection with si-miRNA-339-5p, the expression of miRNA-339-5p, caspase-3, caspase-8 and E-cadherin RNA in the allicin + si-miRNA-339-5p group decreased significantly compared with that in the allicin group, whereas the expression of ZNF-689 and vimentin mRNA increased significantly $(P<0.001$, Figure 8A \& 8B). Western blot analysis (Figure 8C \& 8D) indicated that the expression of caspase-3, caspase-8 and E-cadherin protein in the allicin group and the allicin + si-NC group increased significantly compared with the NC group, whereas the expression of ZNF-689 and vimentin protein decreased significantly $(P<0.001$, Figure 8C \& 8D). After transfection with si-miRNA-339-5p, the expression of caspase-3, caspase- 8 and E-cadherin protein in the allicin + si-miRNA-339-5p group was reduced significantly compared with that in the allicin group, whereas the expression of ZNF-689 and vimentin protein increased significantly $(P<0.001$, Figure 8C \& 8D).

\section{Discussion}

Chemotherapy remains the most effective adjuvant therapy for pancreatic cancer in addition to surgery. However, there are few first- and second-line chemotherapeutic regimens available and the efficacy of these regimens is limited. Therefore, it is imperative to continue to develop new drugs for the treatment of pancreatic cancer. With the discovery of more new drugs, traditional Chinese medicine (TCM) offers certain effective and valuable alternatives to anti-tumour therapeutics. Li et al. [13] showed in a study of first-line an epidermal growth factor receptor tyrosine kinase inhibitor for the treatment of advanced lung adenocarcinoma, adjuvant therapy with TCM significantly reduced the risk of death by $68 \%$ and the risk of disease 
progression by $59 \%$. Additionally, it has been demonstrated that natural plant extracts can effectively inhibit the occurrence and development of tumours [14, 15]. The long-term consumption of garlic can improve immunity and allicin is the main component of garlic extract, which can inhibit the proliferation of various tumour cell types in vitro and in vivo [16-18]. In the present study, the human pancreatic cancer cell lines PANC-1 and Hs766T were treated with allicin at different concentrations. The results indicated that allicin effectively inhibited certain biological activities (proliferation, invasion and migration) of pancreatic cancer cells in a dose-dependent manner.

miRNAs are involved in many important cellular processes. It has been reported that miRNAs can regulate $30 \%$ of human genes and some biological functions, including embryonic development, cell proliferation, apoptosis and differentiation. Abnormal expression of miRNAs may lead to the occurrence of various human diseases, including the aetiology and development of tumours. The mechanisms of miRNA-mediated tumour occurrence and development include acting as oncogenes or tumour suppressor genes and regulating the biological behaviours of tumour cells, such as proliferation, apoptosis, invasion and metastasis. Several studies have demonstrated that miRNA-339-5p can inhibit lung cancer [19, 20], liver cancer [21], breast cancer [22] and leukaemia [23]. In this study, qRT-PCR results indicated that after allicin exposure, the biological activities of pancreatic cancer cell lines (PANC-1 and Hs766T) decreased and the expression of miRNA-339-5p increased significantly. However, after the knockout of miRNA-339-5p, the inhibitory effect of allicin on pancreatic cancer cell lines disappeared. Therefore, we suggest that the inhibitory effect of allicin on pancreatic cancer may be closely related to increased expression of miRNA-339-5p.

Zinc finger proteins (ZNF) are a class of proteins that bind to $\mathrm{Zn}^{2+}$, selectively bind to specific target structures and play an important role in gene expression regulation, cell differentiation and embryonic development [24]. ZNF-689 is a ZNF subtype that is highly expressed in tumour cells [25]. We previously demonstrated that miRNA-339-5p could target and regulate ZNF-689 to inhibit the biological activities 
of pancreatic cancer cells [26]. In the present study, our results suggest that the inhibitory effect of allicin on the biological activities of pancreatic cancer may be related to the targeted regulation of ZNF-689 by miRNA-339-5p. Caspases are a group of proapoptotic proteins that primarily promote apoptosis. They receive apoptotic signals and activate caspase-3 by proteolysis. Caspase- 8 is the most important component of the caspase family and is an apoptosis initiator. It is a major protein of the caspase family that triggers apoptosis in mammalian cells, belongs to the aspartate-specific cysteine proteases and acts as the main effector that causes apoptosis $[27,28]$. After synthesis, it usually exists in cells in the form of an inactive zymogen with a long domain that binds to the caspase-activated complex comprised of death receptors (or intracellular proapoptotic proteins) and adaptor molecules on the cell membrane. In tumours, ZNF-689 is negatively correlated with caspase-3 and caspase- 8 expression [29]. After the overexpression of miRNA-339-5p induced by allicin, the expression of ZNF-689 decreases and the expression of caspase-3 and caspase- 8 increases. These may represent targets of allicin that result in the inhibition of proliferation and promotion of apoptosis in pancreatic cancer cells.

The main cause of death in cancer patients is tumour metastasis, which is often one of the main causes of treatment failure. EMT is a key factor of tumour recurrence and metastasis, during which contact between the epithelial layer and cells results in a loss of polarity, causing the inhibition of cell adhesion. This results in a significant remodelling of the cytoskeleton and increased cell mobility, invasion and metastasis. These events are accompanied by down-regulation of the epithelial marker, E-cadherin and up-regulation of the stromal cell markers, N-cadherin and vimentin $[30,31]$. The expression of ZNF-689 is correlated with the expression of EMT markers (E-cadherin and vimentin) [29]. Our study demonstrated that following allicin treatment, E-cadherin was up-regulated and vimentin was down-regulated, which may be the mechanism that inhibits the invasion and migration of pancreatic cancer cells.

In conclusion, we found that allicin effectively inhibited the biological activities (proliferation, invasion and migration) of pancreatic cancer cells. The mechanism of 
action of allicin may be related to the miRNA-339-5p/ZNF-689 axis and its downstream caspase-3, caspase-8, vimentin and E-cadherin genes. However, there were some limitations to our research. Although we evaluated the anti-tumour effects of allicin in pancreatic cancer cells in vitro, the effect of allicin on pancreatic cancer in vivo should also be determined in future studies.

\section{References:}

1. Siegel RL, Miller KD, Jemal A. Cancer statistics, 2019. CA Cancer J Clin. 2019 Jan; 69(1):7-34.

2. Jorg Kleeff, Murray Korc, Minoti Apte, Carlo La Vecchia, Colin D Johnson, Andrew V Biankin, Rachel E Neale, Margaret Tempero, David A Tuveson, Ralph H Hruban, John P Neoptolemos. Pancreatic cancer. Nat Rev Dis Primers. 2016 Apr 21;2:16022.

3. Pedro Moutinho-Ribeiro, Guilherme Macedo, Sónia A Melo. Pancreatic Cancer Diagnosis and Management: Has the Time Come to Prick the Bubble? Front Endocrinol (Lausanne). 2019 Jan 8; 9:779.

4. Ducreux M, Seufferlein T, Van Laethem JL, Laurent-Puig P, Smolenschi C, Malka D, Boige V, Hollebecque A, Conroy T. Systemic treatment of pancreatic cancer revisited. Semin Oncol. 2019 Feb; 46(1):28-38.

5. Xuecheng Zhang, Yong Zhu, Wei Duan, Chen Feng, Xuan He. Allicin induces apoptosis of the MGC-803 human gastric carcinoma cell line through the p38 mitogen-activated protein kinase/caspase-3 signaling pathway. Mol Med Rep. 2015 Apr; 11(4):2755-60.

6. Bin Song, Ying Shu, Tianlei Cui, Ping Fu. Allicin inhibits human renal clear cell carcinoma progression via suppressing HIF pathway. Int J Clin Exp Med. 2015 Nov 15;8(11):20573-80.

7. Xuejing Zou, Jiyun Liang, Jingyuan Sun, Xiaoyun Hu, Ling Lei, Dehua Wu, Li Liu. Allicin sensitizes hepatocellular cancer cells to anti-tumor activity of 5-fluorouracil through ROS-mediated mitochondrial pathway. J Pharmacol Sci. 2016 Aug; 131(4):233-40. 
8. Huang WL, Wu SF, Xu ST, Ma YC, Wang R, Jin S, Zhou S. Allicin enhances the radiosensitivity of colorectal cancer cells via inhibition of NF-kappaB signaling pathway. J Food Sci. 2020 Jun; 85(6):1924-1931.

9. Frederick Anokye-Danso, Chinmay M Trivedi, Denise Juhr, Mudit Gupta, Zheng Cui, Ying Tian, Yuzhen Zhang, Wenli Yang, Peter J Gruber, Jonathan A Epstein, Edward E Morrisey. Highly efficient miRNA-mediated reprogramming of mouse and human somatic cells to pluripotency. Cell Stem Cell. 2011 Apr 8; 8(4):376-88.

10. Zheng L, Zhang Y, Fu Y, Gong H, Guo J, Wu K, Jia Q, Ding X. Long non-coding RNA MALAT1 regulates BLCAP mRNA expression through binding to miR-339-5p and promotes poor prognosis in breast cancer. Biosci Rep. 2019 Feb 15; 39(2):BSR20181284.

11. Li Y, Zhang X, Yang Z, Li Y, Han B, Chen LA. miR-339-5p inhibits metastasis of non-small cell lung cancer by regulating the epithelial-to-mesenchymal transition. Oncol Lett. 2018 Feb; 15(2):2508-2514.

12. Liu EL, Zhou YX, Li J, Zhang DH, Liang F. Long-Chain Non-Coding RNA SNHG3 Promotes the Growth of Ovarian Cancer Cells by Targeting miR-339-5p/TRPC3 Axis. Onco Targets Ther. 2020 Oct 28; 13:10959-10971.

13. Chia-Ling Li, Te-Chun Hsia, Chia-Hsiang Li, Ko-Jung Chen, Yao-Hsu Yang, Su-Tso Yang. Adjunctive Traditional Chinese Medicine Improves Survival in Patients With Advanced Lung Adenocarcinoma Treated With First-Line Epidermal Growth Factor Receptor (EGFR) Tyrosine Kinase Inhibitors (TKIs): A Nationwide, Population-Based Cohort Study. Integr Cancer Ther. Jan-Dec 2019; 18:1534735419827079.

14. Adiwidjaja J, McLachlan AJ, Boddy AV. Curcumin as a clinically-promising anti-cancer agent: pharmacokinetics and drug interactions. Expert Opin Drug Metab Toxicol. 2017 Sep; 13(9):953-972.

15. Davatgaran-Taghipour Y, Masoomzadeh S, Farzaei MH, Bahramsoltani R, Karimi-Soureh Z, Rahimi R, Abdollahi M. Polyphenol nanoformulations for cancer therapy: experimental evidence and clinical perspective. Int $\mathrm{J}$ Nanomedicine. 2017 Apr 4; 12:2689-2702. 
16. El-Ratel IT, Abdel-Khalek AE, Gabr SA, Hammad ME, El-Morsy HI. Influence of allicin administration on reproductive efficiency, immunity and lipid peroxidation of rabbit does under high ambient temperature. J Anim Physiol Anim Nutr (Berl). 2020 Mar; 104(2):539-548.

17. Pandey N, Tyagi G, Kaur P, Pradhan S, Rajam MV, Srivastava T. Allicin Overcomes Hypoxia Mediated Cisplatin Resistance in Lung Cancer Cells through ROS Mediated Cell Death Pathway and by Suppressing Hypoxia Inducible Factors. Cell Physiol Biochem. 2020 Aug 19; 54(4):748-766.

18. Rosas-González VC, Téllez-Bañuelos MC, Hernández-Flores G, Bravo-Cuellar A, Aguilar-Lemarroy A, Jave-Suárez LF, Haramati J, Solorzano-Ibarra F, Ortiz-Lazareno PC. Differential effects of alliin and allicin on apoptosis and senescence in luminal A and triple-negative breast cancer: Caspase, deltapsim, and pro-apoptotic gene involvement. Fundam Clin Pharmacol. 2020 Dec; 34(6):671-686.

19. Wang J, Jiang M, Xia S. miR-339-5p Increases Radiosensitivity of Lung Cancer Cells by Targeting Phosphatases of Regenerating Liver-1 (PRL-1). Med Sci Monit. 2018 Nov $21 ; 24: 8408-8416$.

20. Gan CZ, Li G, Luo QS, Li HM. miR-339-5p downregulation contributes to Taxol resistance in small-cell lung cancer by targeting alpha1,2-fucosyltransferase 1 . IUBMB Life. 2017 Nov; 69(11):841-849.

21. Wang YL, Chen CM, Wang XM, Wang L. Effects of miR-339-5p on invasion and prognosis of hepatocellular carcinoma. Clin Res Hepatol Gastroenterol. 2016 Feb; 40(1):51-6.

22. Zheng L, Zhang Y, Fu Y, Gong H, Guo J, Wu K, Jia Q, Ding X. Long non-coding RNA MALAT1 regulates BLCAP mRNA expression through binding to miR-339-5p and promotes poor prognosis in breast cancer. Biosci Rep. 2019 Feb 15; 39(2):BSR20181284.

23. Sun X, Liu H, Li T, Qin L. MicroRNA-339-5p inhibits cell proliferation of acute myeloid leukaemia by directly targeting SOX4. Mol Med Rep. 2018 Dec; 18(6):5261-5269. 
24. Jen J, Wang YC. Zinc finger proteins in cancer progression. J Biomed Sci. 2016; 23(1):53.

25. Yi PS, Wu B, Deng DW, Zhang GN, Li JS. Positive expression of ZNF689 indicates poor prognosis of hepatocellular carcinoma. Oncol Lett. 2018; 16(4):5122-5130.

26. Zeqian Yu, Susu Zhao, Lishan Wang, Junying Wang, Jiahua Zhou. miRNA-339-5p Plays an Important Role in Invasion and Migration of Pancreatic Cancer Cells. Med Sci Monit. 2019 Oct 7; 25:7509-7517.

27. Dubey M, Nagarkoti S, Awasthi D, Singh AK, Chandra T, Kumaravelu J, Barthwal MK, Dikshit M. Nitric oxide-mediated apoptosis of neutrophils through caspase-8 and caspase-3-dependent mechanism. Cell Death Dis. 2016 Sep 1; 7(9):e2348.

28. Jiao C, Chen W, Tan X, Liang H, Li J, Yun H, He C, Chen J, Ma X, Xie Y, Yang BB. Ganoderma lucidum spore oil induces apoptosis of breast cancer cells in vitro and in vivo by activating caspase-3 and caspase-9. J Ethnopharmacol. 2020 Jan 30; $247: 112256$.

29. Hui Zeng, Jiaping Zheng, Song Wen, Jun Luo, Guoliang Shao, Yongjun Zhang. MicroRNA-339 inhibits human hepatocellular carcinoma proliferation and invasion via targeting ZNF689. Drug Des Devel Ther. 2019 Jan 22; 13:435-445.

30. Yamashita N, Tokunaga E, Iimori M, Inoue Y, Tanaka K, Kitao H, Saeki H, Oki E, Maehara Y. Epithelial Paradox: Clinical Significance of Coexpression of E-cadherin and Vimentin With Regard to Invasion and Metastasis of Breast Cancer. Clin Breast Cancer. 2018 Oct; 18(5):e1003-e1009.

31. Wang JH, Lee EJ, Ji M, Park SM. HDAC inhibitors, trichostatin A and valproic acid, increase E-cadherin and vimentin expression but inhibit migration and invasion of cholangiocarcinoma cells. Oncol Rep. 2018 Jul; 40(1):346-354.

Figure legends

Figure 1. Effect of allicin on proliferation of pancreatic cancer cells

NC: The cell were treated with normal; Low: The cell were treated with low-dose 
Allicin $(5 \mu \mathrm{g} / \mathrm{mL})$; Middle: The cell were treated with middle-dose Allicine $(10 \mu \mathrm{g} / \mathrm{mL})$; High: The cell were treated with high-dose Allicine $(20 \mu \mathrm{g} / \mathrm{mL})$
A. Cell proliferation rate of different PANC-1 cell groups
B. Cell proliferation rate of different Hs766T cell groups
C. EDU-positive PANC-1 cell number $(200 \times)$
D. EDU-positive Hs766T cell number (200×)

*: $\mathrm{P}<0.05, * *: \mathrm{P}<0.01, * * *: \mathrm{P}<0.001$, compared with NC group; \#: $\mathrm{P}<0.05$, \#\#: $\mathrm{P}$ $<0.01$, compared with Low groups; \$: $\mathrm{P}<0.05$, compared with Middle group

Figure 2. Effect of allicin on apoptosis of pancreatic cancer cells

NC: The cell were treated with normal; Low: The cell were treated with low-dose Allicin $(5 \mu \mathrm{g} / \mathrm{mL})$; Middle: The cell were treated with middle-dose Allicine $(10 \mu \mathrm{g} / \mathrm{mL})$; High: The cell were treated with high-dose Allicine $(20 \mu \mathrm{g} / \mathrm{mL})$
A. Apoptosis rate in PANC-1 cell groups
B. Apoptosis rate in Hs766T cell groups
C. TUNEL cell number in PANC-1 cell groups $(100 \times)$
D. TUNEL cell number in Hs766T cell groups $(100 \times)$

*: $\mathrm{P}<0.05, * *: \mathrm{P}<0.01, * * *: \mathrm{P}<0.001$, compared with NC group; \#: $\mathrm{P}<0.05$, \#\#: $\mathrm{P}$ $<0.01$, compared with Low groups; $\$$ : $\mathrm{P}<0.05$, compared with Middle group

Figure 3. Effects of allicin on invasion and migration of pancreatic cancer cells NC: The cell were treated with normal; Low: The cell were treated with low-dose Allicin $(5 \mu \mathrm{g} / \mathrm{mL})$; Middle: The cell were treated with middle-dose Allicine $(10 \mu \mathrm{g} / \mathrm{mL})$; High: The cell were treated with high-dose Allicine $(20 \mu \mathrm{g} / \mathrm{mL})$
A. Invasion PANC-1 cell number $(200 \times)$
B. Invasion Hs766T cell number $(200 \times)$
C. Wound healing rate of different PANC-1 cell groups $(100 \times)$
D. Wound healing rate of different Hs766T cell groups $(100 \times)$

*: $\mathrm{P}<0.05, * *: \mathrm{P}<0.01, * * *: \mathrm{P}<0.001$, compared with $\mathrm{NC}$ group; \#: $\mathrm{P}<0.05$, \#\#: $\mathrm{P}$ 
$<0.01$, compared with Low groups; $\$$ : $\mathrm{P}<0.05$, compared with Middle group

Figure 4. Effects of allicin on relevant genes and proteins

NC: The cell were treated with normal; Low: The cell were treated with low-dose Allicin $(5 \mu \mathrm{g} / \mathrm{mL})$; Middle: The cell were treated with middle-dose Allicine $(10 \mu \mathrm{g} / \mathrm{mL})$; High: The cell were treated with high-dose Allicine $(20 \mu \mathrm{g} / \mathrm{mL})$

A. Relative gene expression in different PANC-1 cell groups

B. Relative gene expression in Hs766T cell groups

C. Relative protein expression in PANC-1 cell groups

D. Relative protein expression in Hs766T cell groups

*: $\mathrm{P}<0.05, * *: \mathrm{P}<0.01, * * *: \mathrm{P}<0.001$, compared with NC group; \#: $\mathrm{P}<0.05$, \#\#: $\mathrm{P}$ $<0.01$, compared with Low groups; \$: $\mathrm{P}<0.05$, compared with Middle group

Figure 5. Effect of miRNA-339-5p in allicin inhibiting proliferation of pancreatic cancer cells

NC: The cell were treated with normal; Allicin: The cell were treated with Allicin $(20 \mu \mathrm{g} / \mathrm{mL}) ;$ Allicin+si-NC: The cell were transfection with si-NC (negative control) and treated with Allicin $(20 \mu \mathrm{g} / \mathrm{mL})$; Allicin+si-miRNA-339-5p: The cell were transfected with si-miRNA-339-5p and treated with Allicin $(20 \mu \mathrm{g} / \mathrm{mL})$
A. Cell proliferation rate of different PANC-1 cell groups
B. Cell proliferation rate of different Hs766T cell group
C. EDU-positive PANC-1 cell number $(200 \times)$
D. EDU-positive Hs766T cell number(200×)

***: $\mathrm{P}<0.001$, compared with $\mathrm{NC}$ group; \#\#\#: $\mathrm{P}<0.001$, compared with Allicin group

Figure 6. Effect of miRNA-339-5p in allicin promoting apoptosis of pancreatic cancer cells

NC: The cell were treated with normal; Allicin: The cell were treated with Allicin $(20 \mu \mathrm{g} / \mathrm{mL}) ;$ Allicin+si-NC: The cell were transfection with si-NC (negative control) 
and treated with Allicin $(20 \mu \mathrm{g} / \mathrm{mL})$; Allicin+si-miRNA-339-5p: The cell were transfected with si-miRNA-339-5p and treated with Allicin $(20 \mu \mathrm{g} / \mathrm{mL})$
A. Apoptosis rate in PANC-1 cell groups
B. Apoptosis rate in Hs766T cell groups
C. TUNEL cell number in PANC-1 cell groups $(100 \times)$
D. TUNEL cell number of Hs766T cell groups $(100 \times)$

***: $\mathrm{P}<0.001$, compared with $\mathrm{NC}$ group; \#\#\#: $\mathrm{P}<0.001$, compared with Allicin group

Figure 7. Effects of miRNA-339-5p in allicin inhibiting invasion and migration of pancreatic cancer cells

NC: The cell were treated with normal; Allicin: The cell were treated with Allicin $(20 \mu \mathrm{g} / \mathrm{mL}) ;$ Allicin+si-NC: The cell were transfection with si-NC (negative control) and treated with Allicin $(20 \mu \mathrm{g} / \mathrm{mL})$; Allicin+si-miRNA-339-5p: The cell were transfected with si-miRNA-339-5p and treated with Allicin $(20 \mu \mathrm{g} / \mathrm{mL})$
A. Invasion PANC-1 cell number $(200 \times)$
B. Invasion Hs766T cell number $(200 \times)$
C. Wound healing rate of different PANC-1 cell groups $(100 \times)$
D. Wound healing rate of different Hs766T cell groups $(100 \times)$

***: $\mathrm{P}<0.001$, compared with NC group; \#\#\#: $\mathrm{P}<0.001$, compared with Allicin group

Figure 8. Expressions of relevant genes and proteins

NC: The cell were treated with normal; Allicin: The cell were treated with Allicin $(20 \mu \mathrm{g} / \mathrm{mL})$; Allicin+si-NC: The cell were transfection with si-NC (negative control) and treated with Allicin $(20 \mu \mathrm{g} / \mathrm{mL})$; Allicin+si-miRNA-339-5p: The cell were transfected with si-miRNA-339-5p and treated with Allicin $(20 \mu \mathrm{g} / \mathrm{mL})$
A. Relative gene expression in PANC-1 cell groups
B. Relative gene expression in Hs766T cell groups
C. Relative protein expression in PANC-1 cell groups 
D. Relative protein expression in Hs766T cell groups

***: $\mathrm{P}<0.001$, compared with NC group; \#\#\#: $\mathrm{P}<0.001$, compared with Allicin group 
Table 1 . The primer sequence

\begin{tabular}{ccc}
\hline Gene Name & F:(5'-3') & R:(5'-3') \\
\hline miRNA-339-5p & ACACTCCAGCTGCGGTCCCTGTCCTCCAGGAG & TGGTGTCGTGGAGTCG \\
ZNF-689 & TGGAACGAAACACCGATGACT & CCATTCTTCTTTCTGGTTCTGCT \\
Vimentin & GACGCCATCAACACCGAGTT & CTTTGTCGTTGGTTAGCTGGT \\
E-cadherin & ACAGGATGGCTGAAGGTGAC & GGATGACACAGCGTGAGAGA \\
Caspase-3 & CAGACAGTGGAACTGACGAT & TTTCAGCATGGCGCAAAGTG \\
Caspase-8 & CGCAAAGGAAGACAAGAAC & TTGAGCCCTGCCTGGTGT \\
U6 & CTCGCTTCGGCAGCACA & AACGCTTCACGAATTTGCT \\
GAPDH & GGTGAAGGTCGGTGTGAACG & GCTCCTGGAAGATGGTGATGG \\
\hline
\end{tabular}




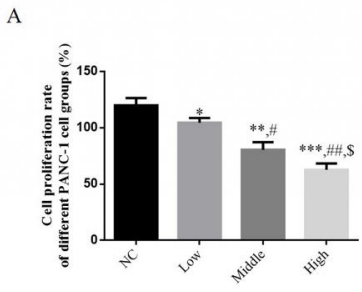

C
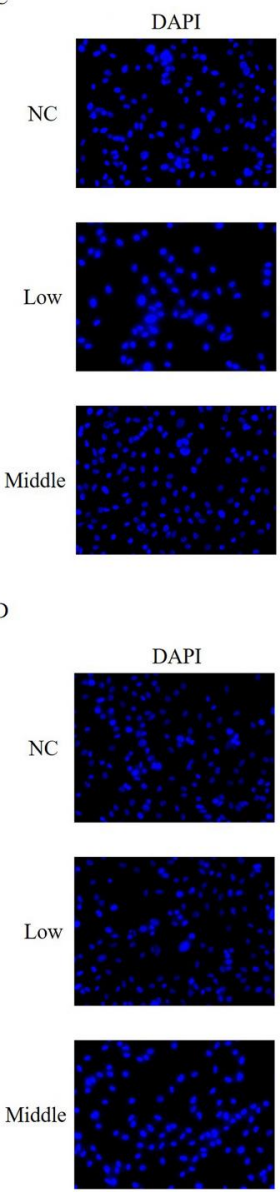
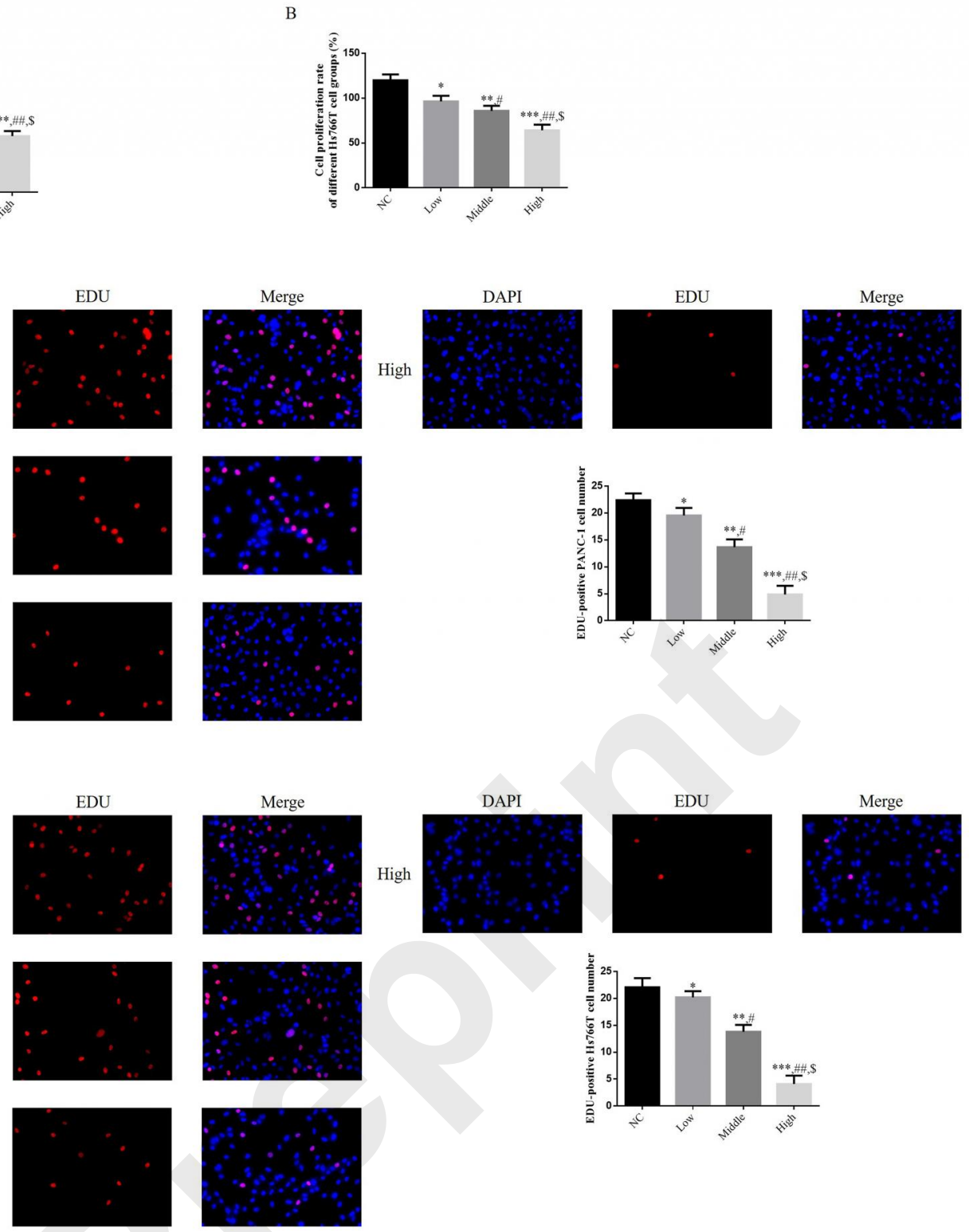

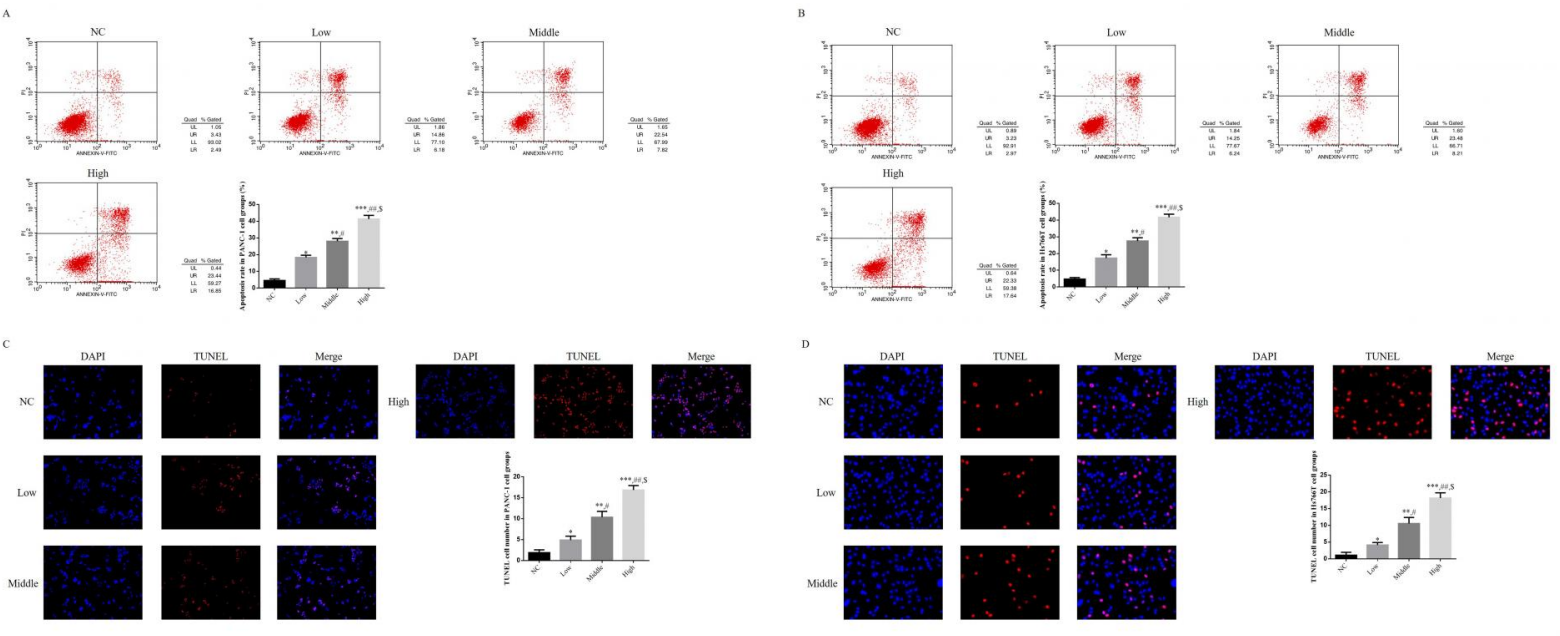

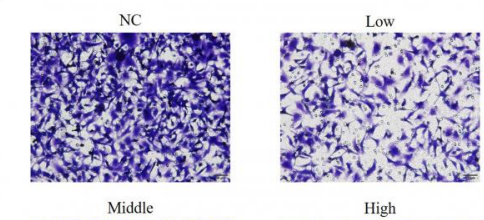

X Middle

-1) 310

$10 \mathrm{cos}$
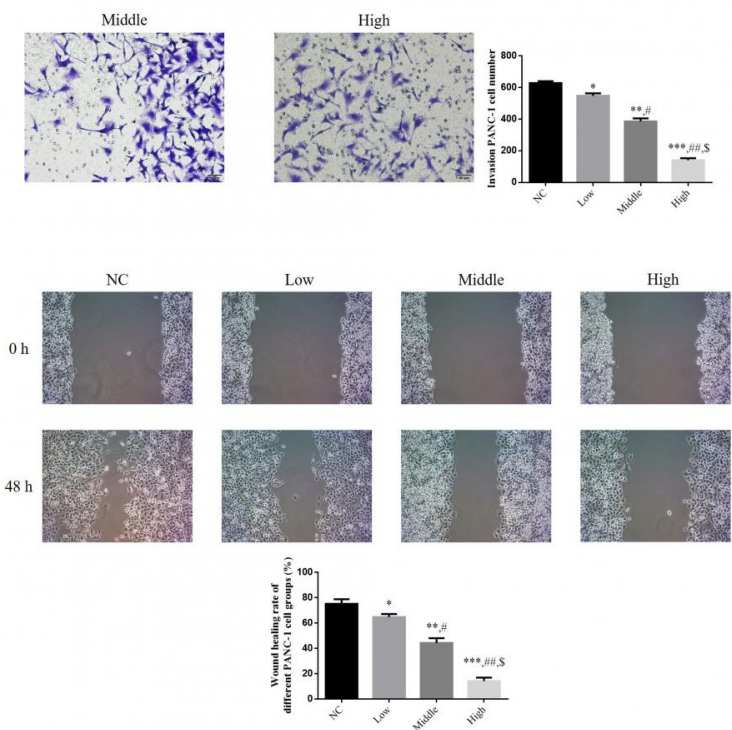
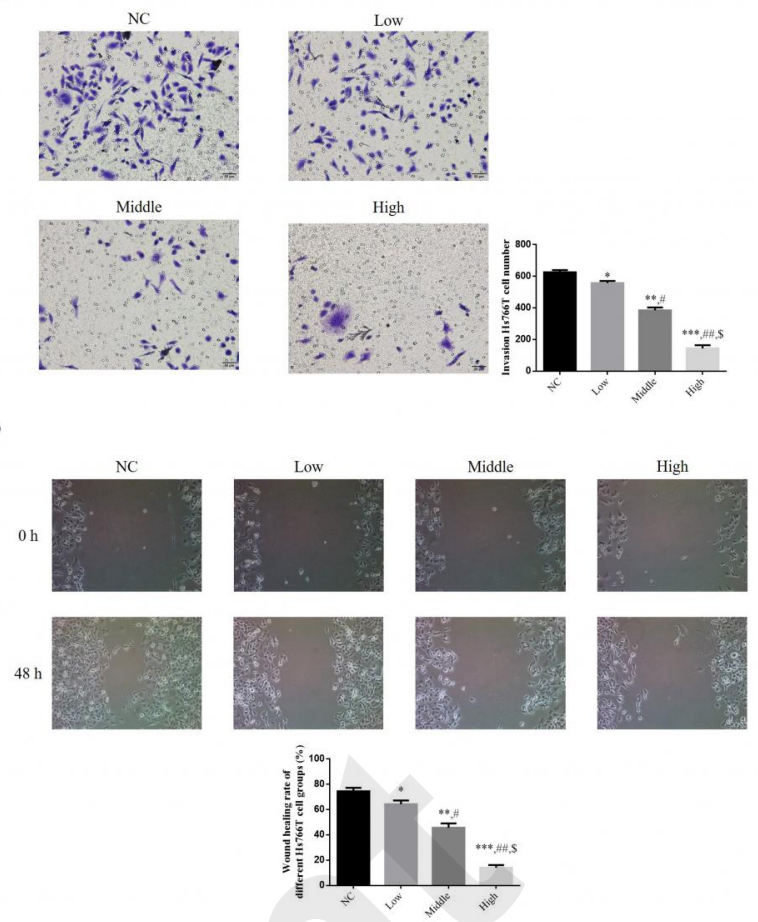

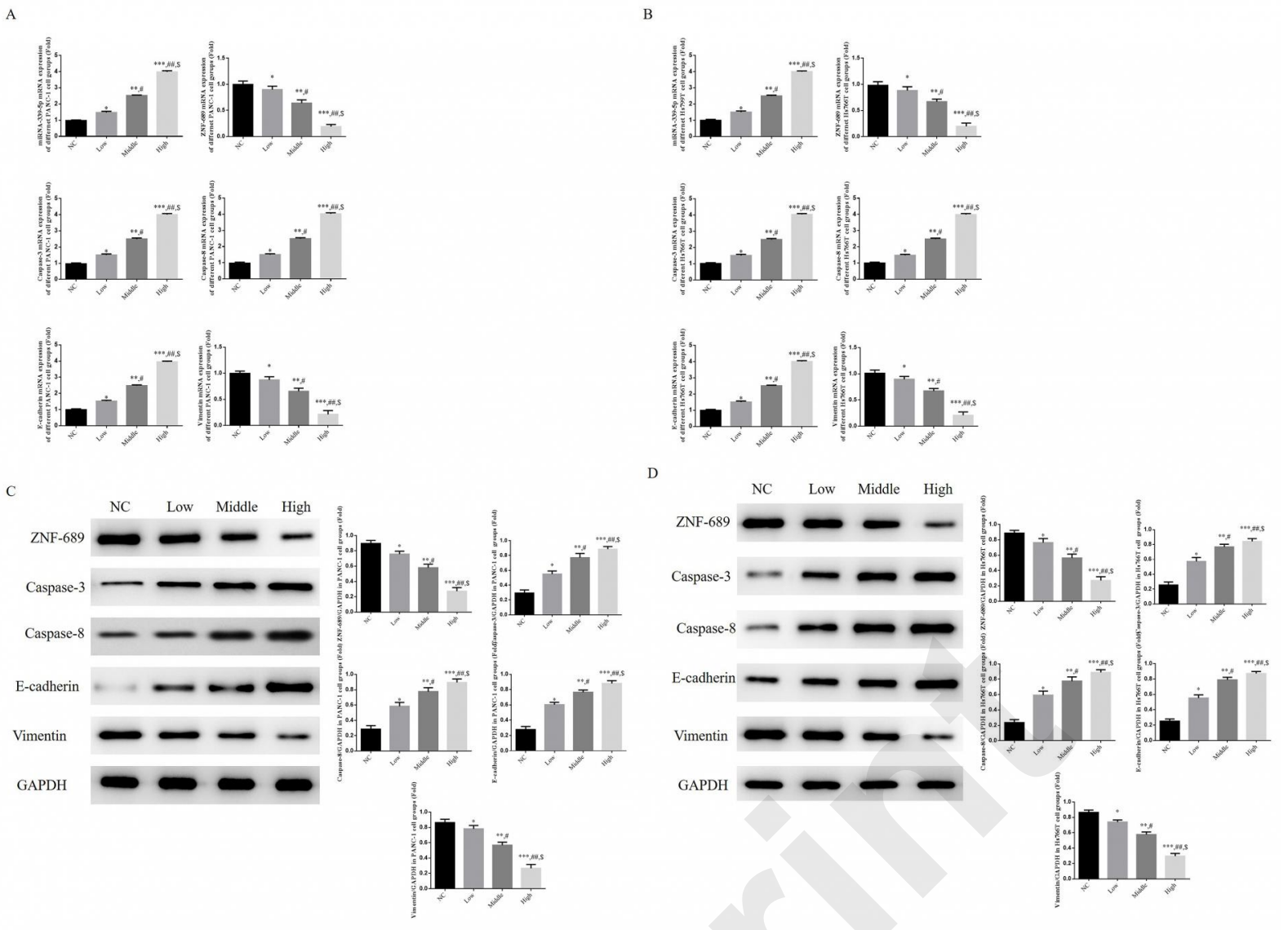

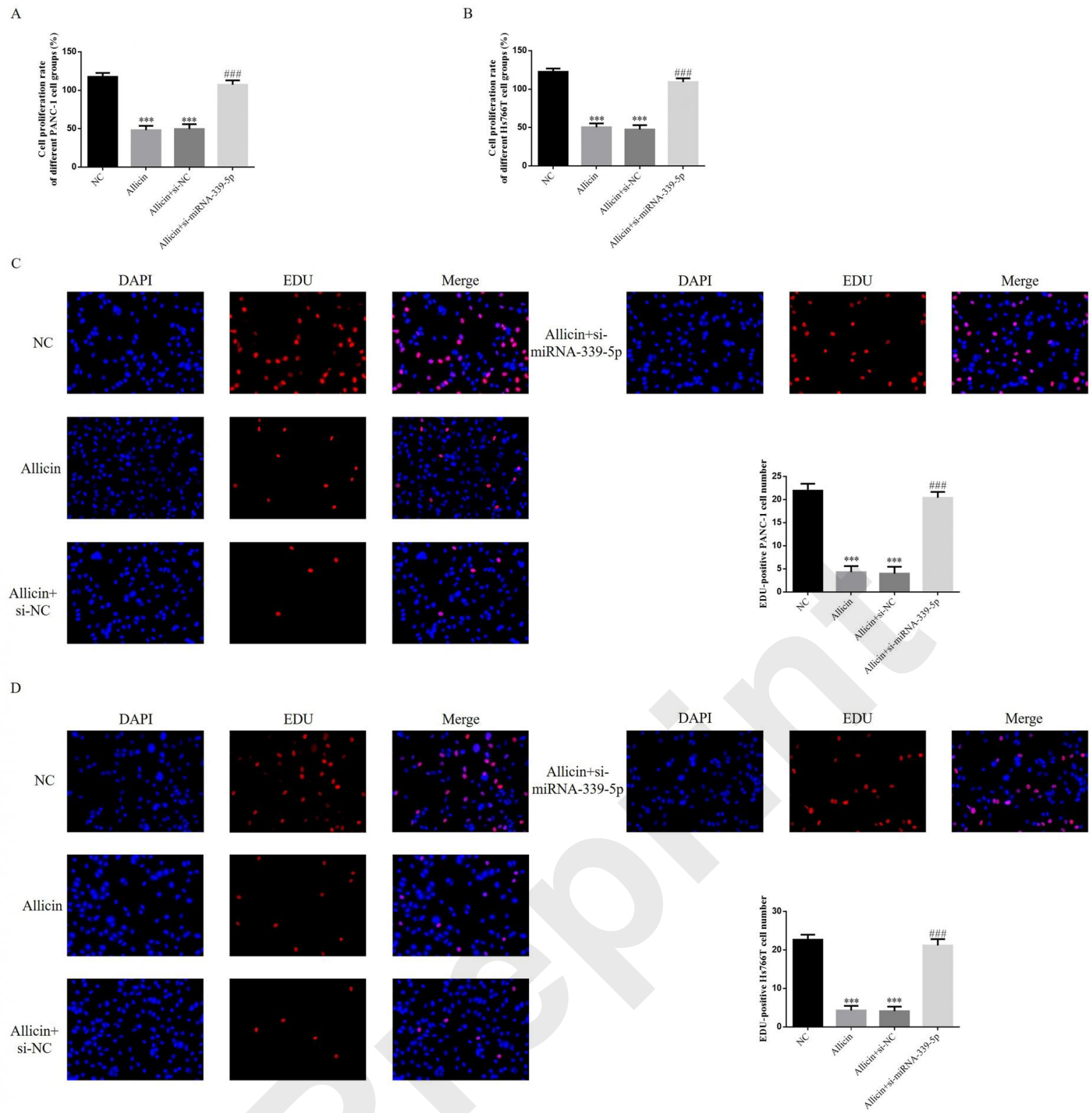


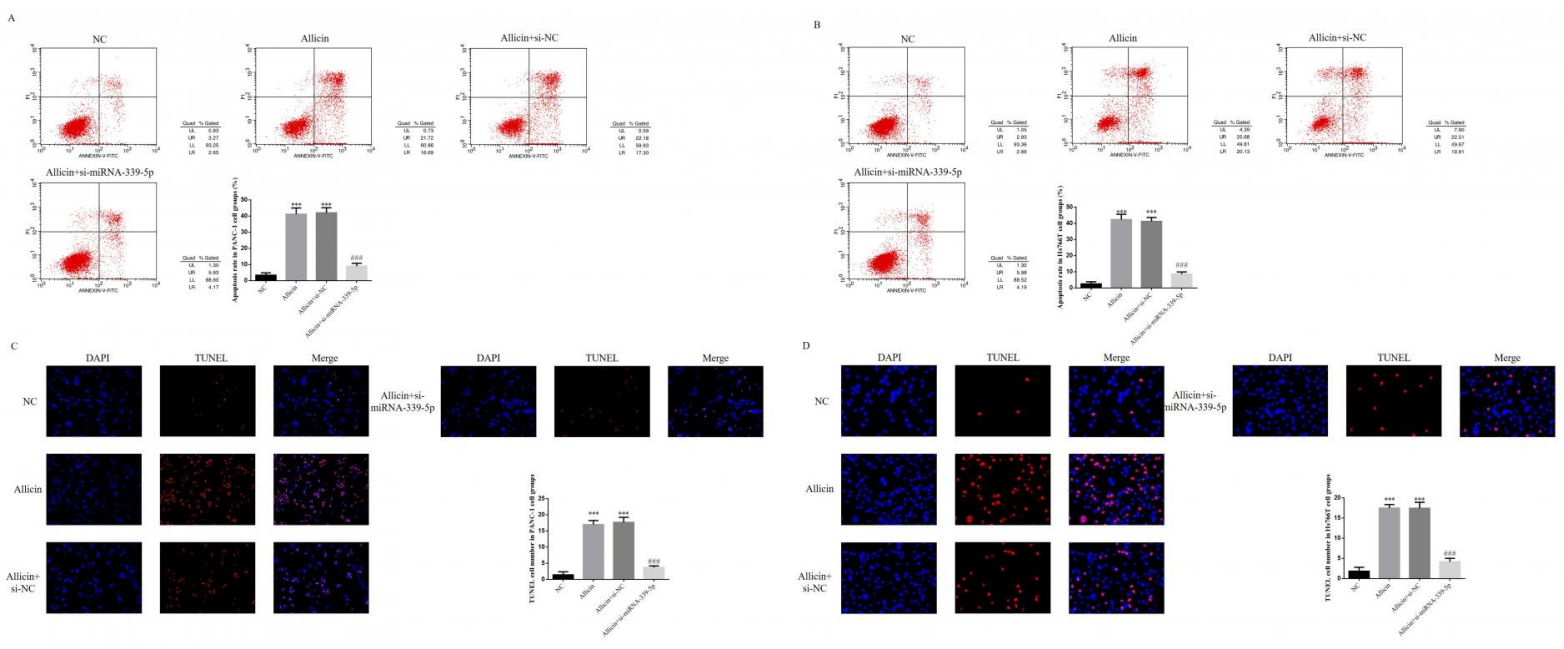



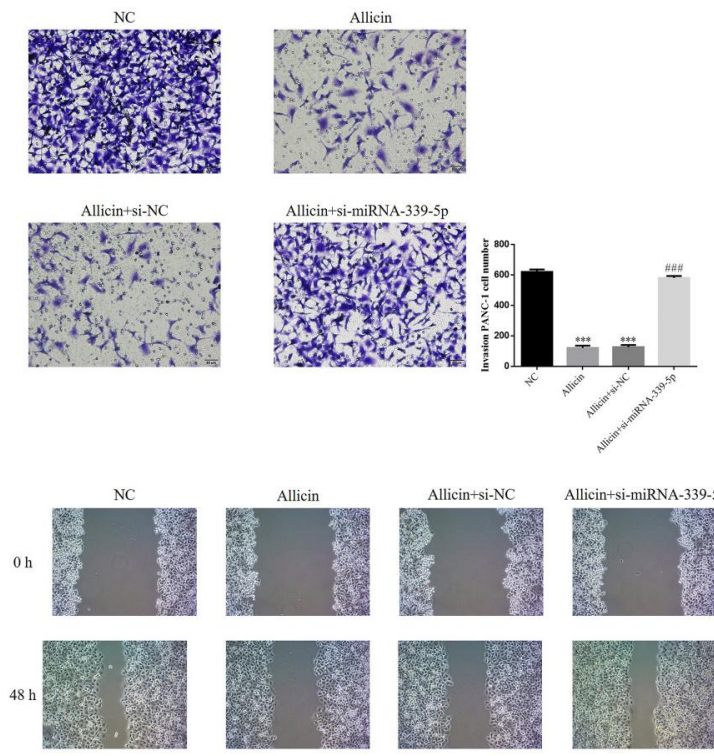

(1)
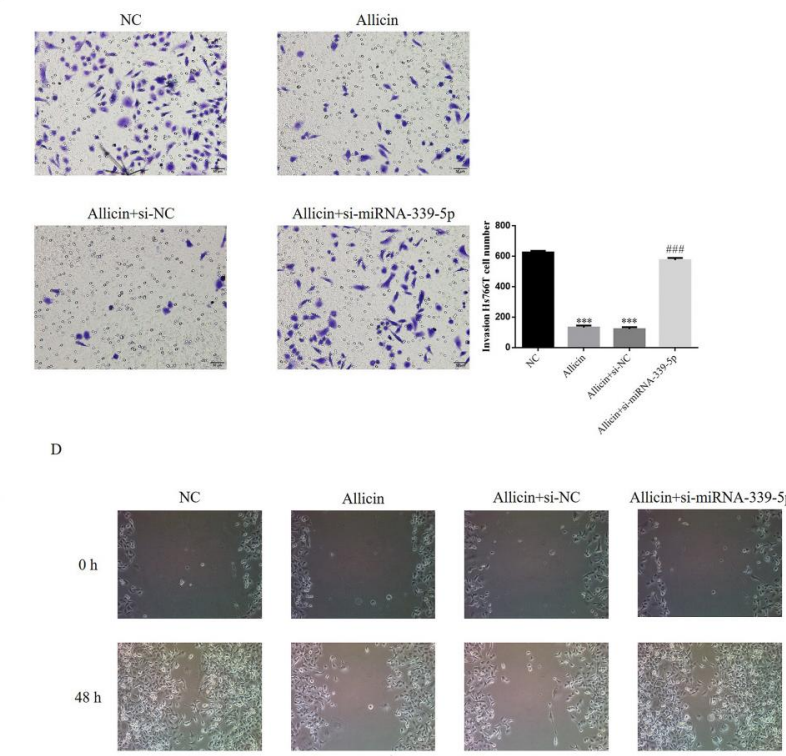

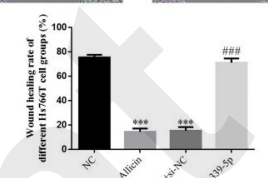


In:

$\lim _{2}$

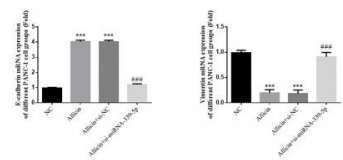

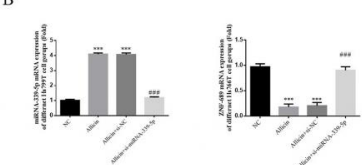

$\lim _{2}$

U:

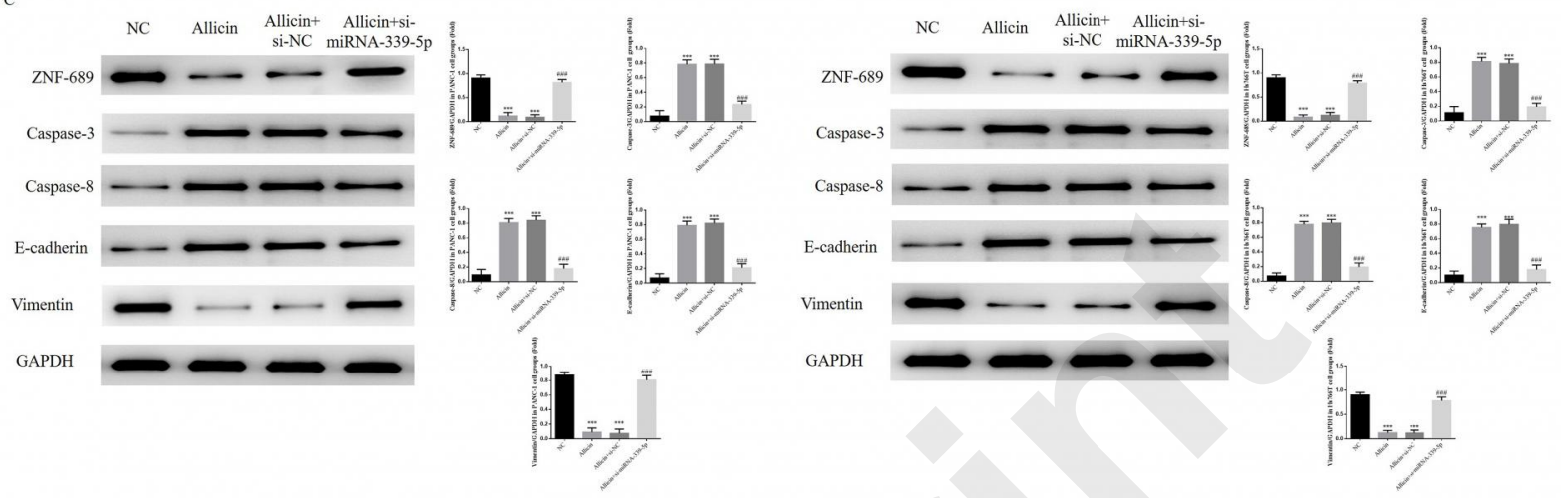

